\title{
Reversible cerebral ischemia in patients with pheochromocytoma
}

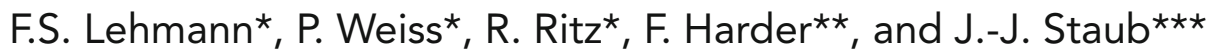 \\ ${ }^{*}$ Intensive Care Unit, Department of Internal Medicine, ${ }^{\star \star}$ Department of Surgery, ${ }^{* \star *}$ Division of \\ Endocrinology, University Hospital of Basel, Switzerland
}

\begin{abstract}
Cerebral ischemia and symptoms of stroke can occur as a rare manifestation in patients with pheochromocytoma. We describe a 45-year-old woman who was admitted because of a right-sided hemiparesis due to an ischemic lesion in the left hypothalamus. The clinical diagnosis of a pheochromocytoma was proven by highly elevated urinary catecholamines and confirmed histologically after operation. The successful removal of the tumor led to the almost
\end{abstract}

\section{INTRODUCTION}

Pheochromocytoma is a rare tumour which occurs approximately in $0.13 \%$ of the general population (1). The typical manifestations of pheochromocytoma are paroxysmal or sustained hypertension with sudden attacks of sweating, palpitations, tremor and headache (2). Neurological symptoms in patients with pheochromocytoma have been reported (3). Reversible cerebral ischemia with focal neurological deficiencies is a rare manifestation of pheochromocytoma. We observed a patient with neurological defects as presenting symptoms of a phaeochromocytoma.

\section{CASE REPORT}

A 45-year-old woman was admitted because of a gradual onset of right-sided hemiparesis. On neurological examination the patient was alert and oriented, but she had a sensomotoric hemiparesis, which affected mainly the upper limb. We observed a central facial palsy as well as hyperreflexia and a

Key-words: Pheochromocytoma, cerebral, ischemia, neurological.

Correspondence: Jean-Jacques Staub, M.D., Professor of Endocrinology, Division of Endocrinology, Dept. of Medicine, University Hospital of Basel, 4031, Basel, Switzerland.

Accepted October 1, 1998 complete recovery of the neurological deficiencies. It is of vital importance to know this atypical presentation of pheochromocytoma. The diagnosis of pheochromocytoma should be suspected in patients with focal cerebral symptoms, particularly in the presence of intermittent hypertension or other paroxysmal symptoms suggestive of pheochromocytoma.

(J. Endocrinol. Invest. 22: 212-214, 1999)

๑1999, Editrice Kurtis

positive sign of Babinski on the right side. On admission the blood pressure was normal (120/70 $\mathrm{mmHg}$ ) with no history of hypertension. The CT scan revealed a small hypodense lesion in the left hypothalamus. Extensive diagnostic evaluation failed to show a cause for this lesion except a ventricular arrythmia, Lown grade IVa. A therapy with sotalol $160 \mathrm{mg}$ daily was begun. The patient recovered and left the hospital after 17 days. On discharge, all motor deficiencies had resolved and the patient showed only slight numbness and dysmetria of her right arm. These symptoms persisted in the long-term follow-up.

At home, repeated attacks of sweating, dizziness, nausea and one syncope occurred under sotalol and she was readmitted to the intensive care unit. This was the first time, when symptoms suggesting catecholamine hypersecretion were present. A prolonged QT interval was observed and an episode of torsade de pointes with consecutive ventricular fibrillation occurred. After resuscitation a blood pressure of 260/159 $\mathrm{mmHg}$ was measured. The diagnosis of a pheochromocytoma was established by the detection of very high levels of urinary catecholamines: Norepinephrine 16,165 nmol/die (normal up to 10) and epinephrine $4308 \mathrm{nmol} /$ die (normal up to 110) and a right-sided, large adrenal mass of $8 \times 10 \mathrm{~cm}$ in the CT scan (Fig. 1). Preoperative adrenergic blockade was established by phenoxy- 


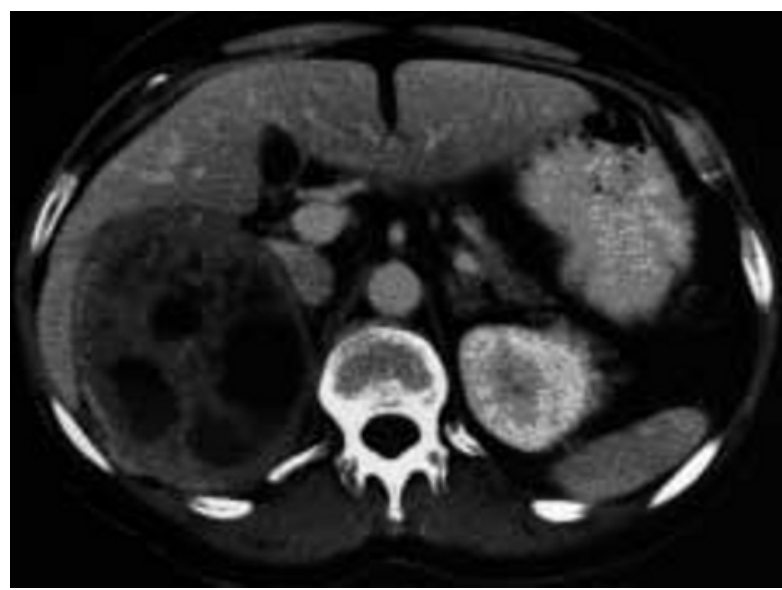

Fig. 1 - Enhanced abdominal CT scan reveals a large $(8 \times 10 \mathrm{~cm})$ right adrenal mass with areas of necrosis and haemorrhagic fluid.

benzamine and propranolol for 10 days. An anterior transabdominal approach was first performed to remove the tumour. However, the upper pole of the pheochromocytoma could not be detached adequately and an additional transthoracic approach was necessary. Manipulation of the tumor induced acute hypertension, that could be effectively treated by sodium nitroprusside and phentolamin. The tumor could be removed successfully and the histological examination confirmed the clinical diagnosis of a benign pheochromocytoma of $600 \mathrm{~g}$ weight.

\section{DISCUSSION}

The clinical presentation of our patient is unusual because of the neurological presenting symptoms leading to hospital admission and because the neurological symptoms were almost completely reversible. Only few reports exist about the occurrence of an ischemic cerebral infarction in patients with pheochromocytoma $(1,3)$. Some of them were at least partially reversible. Thomas et al. described transient neurological symptoms in 11 and cerebral infarctions in 3 of 100 patients with pheochromocytoma (4).

There are several reasons for the onset of an acute ischemic cerebral infarction in patients with pheochromocytoma. Hypertension alone can result in cerebral infarction in patients with pheochromocytoma as published recently by Goswami et al. (5). Hypertensive encephalopathy may be caused by a failure of cerebral vessel autoregulation with very high blood pressure values and can occur with a single crisis of hypertension without chronic hypertension (3). In our patient, the paroxysmal hypertension was observed only after treatment with sotalol was introduced. The paradoxical rise in blood pressure in patients with pheochromocytoma may supervene with administration of $\beta$-blockers, revealing unopposed $\alpha$-adrenoreceptor stimulation. However, the pathological changes of hypertensive encephalopathy consist of widespread small areas of ischemic injury and are not restricted to the distribution of single vessels $(3,5)$. A paroxysm of hypertension may be followed by prolonged hypotension due to systemic exhaustion of catecholamines (3). Systemic hypotension produces either widespread microscopic lesions or larger infarctions in the cerebral border zones (3).

The brain lesions may have been caused by spasms of the cerebral arteries, since vascular spasms in peripheral, coronary and cerebral arteries have been induced with sympathomimetic agents (6) and have also been reported in patients with pheochromocytoma $(3,7)$. Spasm produces either transient impairment of circulation or infarction (8). Cerebral vasospasm can be induced either by excessive catecholamines (5) or by other secretory products of the adrenal tumor, such as neuropeptide $Y$, renin, endothelin or angiotensin-converting enzyme (9). These substances are not only vasoactive but can also promote platelet aggregation $(10,11)$.

Cerebral ischemia and symptoms of stroke can occur as presenting features in some patients with pheochromocytoma. It is of vital importance to know this atypical presentation and to establish early diagnosis in order to avoid irreversible neurological damage or death in such patients. We conclude that the diagnosis of pheochromocytoma should always be suspected in patients with focal cerebral symptoms, particularly in the presence of intermittent hypertension or other paroxysmal symptoms suggestive of pheochromocytoma.

\section{REFERENCES}

1. Fox J.M., Manninen P.H.

The anaesthetic management of a patient with a pheochromocytoma and acute stroke. Can. J. Anaesth. 38: 775, 1991.

2. Ross E.J., Griffith D.N.W. The clinical presentation of pheochromocytoma. Q. J. Med. 266: 485, 1989.

3. Scully R.E., Mark E.J., McNeely W.V., McNeely B.U. Case records of the Massachusetts General Hospital, Case 15-1988.

N. Engl. J. Med. 318: 970, 1988.

4. Thomas J.E., Rooke E.D., Kvale W.F. The neurologist's experience with pheochromocytoma. JAMA 197: 754, 1966. 
5. Goswami R., Tandon N., Singh B., Kochupillai N. Adrenal tumour, congestive heart failure and hemiparesis in an 18-year-old male. A clinical-pathological conference.

Int. J. Cardiol. 49: 233, 1995.

6. Isner J.M., Estes N.A.M., Thompson P.D. Acute cardiac events temporally related to cocaine abuse.

N. Engl. J. Med. 315: 1438, 1986.

7. Sardesai S.H., Mourant A.J., Sivathandon Y., Farrow B., Gibbons D.O.

Pheochromocytoma and catecholamine-induced cardiomyopathy presenting as heart failure. Br. Heart J. 63: 234, 1990.

8. White P.D., Lishman W.A., Wyke M.A.
Pheochromocytoma as a cause of reversible dementia.

J. Neurol. Neurosurg. Psychiatry 49: 1449, 1986.

9. Fonseca V., Bouloux P.M.

Phaeochromocytoma and paraganglioma.

Clin. Endocrinol. Metab. 7: 509, 1993.

10. Zukowska-Grojec Z.

Neuropeptide Y. A novel sympathetic stress hormone and more.

Ann. NY Acad. Sci. 771: 219, 1995.

11. Kirsten R., Breidert M., Sparwasser K., Ochs J.G., Hesse K., Nelson K.

Carmoxirole inhibits platelet aggregation in vitro and ex vivo.

Int. J. Clin. Pharmacol. Ther. 33: 76, 1995. 